\title{
Accesibilidad geográfica a los servicios de salud: un estudio de caso para Barranquilla
}

Geographical Accessibility to Health Services: A Case Study for Barranquilla

Acessibilidade geográfica aos serviços de saúde: um estudo de caso para Barranquilla

\section{Gerson Javier Pérez Valbuena'}

Investigador del Centro de Estudios Económicos Regionales (CEER) del Banco de la República, Cartagena-Colombia

gjavierperezv@gmail.com 


\title{
Resumen
}

En este artículo se utiliza la ampliación de la red pública hospitalaria de Barranquilla para evaluar los avances en la accesibilidad geográfica y la equidad en la prestación del servicio de salud. Se emplean medidas de gravedad para calcular la accesibilidad considerando restricciones a la movilidad, así como información de demanda por servicios de salud. Adicionalmente, se utilizan indicativos de satisfacción para evaluar los niveles de equidad logrados con la ampliación de la red pública. Los resultados muestran avances significativos en accesibilidad geográfica entre 2008 y 2013, especialmente para los habitantes de las localidades más pobres. En términos de equidad, se encontró que los factores socioeconómicos siguen siendo determinantes de la satisfacción de los usuarios del sistema de salud.

Palabras clave: Economía de la Salud, Accesibilidad Espacial, Modelos de Umbral Generalizados.

Clasificación JEL: I18, R53, C35.

\begin{abstract}
In this article, the expansion of the public hospital network in Barranquilla in 2008 is used to assess improvements in geographical accessibility and equality in providing health care services. Particularly, seriousness measures are used to calculate accessibility, considering mobility restrictions, as well as information regarding demand for health care services. Additionally, data on satisfaction indicators is used to assess levels of equality achieved with the expansion of the expansion of the public health care network. The results show significant improvements in accessibility between 2008 and 2013, especially within the poorest locations. In terms of equality, it was found that satisfaction with health services still depends on social-economic factors.
\end{abstract}

Keywords: Health Economics; Spatial Accessibility; Generalized Threshold Models.

\section{Resumo}

Este artigo utiliza a ampliação da rede pública hospitalar de Barranquilla, para avaliar os avanços da acessibilidade geográfica e da equidade na prestação de serviços de saúde. Empregam-se medidas de gravidade para calcular a acessibilidade, tendo em vista restrições na mobilidade e a informação da demanda por serviços de saúde. Além do mais, usa-se indicadores de satisfação para avaliar os níveis de equidade atingidos com dita ampliação. Os resultados evidenciam avanços significativos na acessibilidade geográfica entre 2008 e 2013 , especialmente para os habitantes mais pobres. No que respeita à equidade, encontrou-se que os fatores socioeconómicos seguem sendo determinantes na satisfação dos usuários do sistema de saúde.

Palavras-chave: Economia da Saúde, Acessibilidade Espacial, Modelos com Patamar. 


\section{Introducción}

En todas las ciudades, el acceso a los servicios de salud de la red pública hospitalaria ha sido un asunto de la mayor importancia, especialmente a los servicios de primer nivel, ya que implican menores costos para los gobiernos locales, son de fácil prestación y, por su carácter preventivo, resultan mucho más efectivos en el largo plazo (Guagliardo 2004). Para los condados de Carolina del Sur, Estados Unidos, Hossain y Laditka (2009) encontraron que son precisamente las personas que menor acceso tienen al servicio de salud de primer nivel, las que muestran una mayor tasa de visitas a las salas de emergencia de los hospitales. Sin embargo, a pesar de los claros beneficios sociales que un mayor acceso significa, los gobiernos locales se ven enfrentados a restricciones presupuestales, especialmente por los altos costos que implica mejorar el acceso en comunidades relativamente aisladas o con baja accesibilidad.

Los recientes avances en términos de los sistemas de información geográfica (SIG) han abierto nuevas oportunidades para el análisis y la modelación de medidas de accesibilidad, lo cual es útil en la focalización de políticas de desarrollo en las áreas geográficas con baja provisión de servicios, o con evidentes niveles de inequidad (Brabyn y Skelly 2002; Christie y Fone 2003). Igual que este documento, la mayoría de trabajos analizan la accesibilidad a la red pública de las áreas urbanas; Gibson et al. (2011) presenta una primera aproximación de la distancia a la que se encuentran los hogares para el caso de la provincia de Shaanxi, China.

La principal contribución de este documento es que ofrece, para un área urbana en Colombia, una discusión formal acerca del acceso geográfico a los recursos públicos urbanos, y cómo los gobiernos locales pueden, mediante la distribución geográfica de estos recursos, mantener niveles adecuados de los servicios a su cargo, tales como la salud y la educación. Este documento se basa en el aumento, a partir de 2009, del número de Instituciones Prestadoras de Servicio (IPS) en Barranquilla como un cuasi-experimento que tiene el objetivo de analizar los efectos sobre la accesibilidad absoluta y relativa a la red pública hospitalaria utilizando datos georreferenciados. De igual forma, gracias a la información de las encuestas de satisfacción con el servicio recibido por parte de los usuarios, se presentan los primeros resultados en términos de equidad a través de modelos de elección discreta.

Los resultados dejan ver mejoras sustanciales en términos de accesibilidad geográfica de los usuarios, especialmente los de menores recursos, reduciendo en forma importante la distancia que deben recorrer para recibir un servicio tanto prioritario como especializado. Adicionalmente, los barranquilleros manifiestan los más altos niveles de satisfacción (dentro del grupo de las principales ciudades), no solo en cuanto a los servicios de salud que reciben sino acerca de la imagen que tienen de la Secretaría de Salud del Distrito. En términos de los niveles de desigualdad en la prestación del servicio, se encontró que la ciudad no es la más desigual comparada con ciudades de desarrollo semejante, pero aún se requieren esfuerzos adicionales para que la situación socioeconómica no sea un factor determinante en el nivel de satisfacción con la prestación del servicio. 
Este documento está organizado de la siguiente manera. La primera sección está dedicada a presentar el nuevo modelo de prestación de servicios de salud en Barranquilla, a través del cual fue ampliada la red pública hospitalaria a partir de 2009. En la segunda se muestran los indicadores de cobertura y capacidad instalada antes y después de la implementación del nuevo modelo de prestación de servicios en donde se observa la nueva reorganización espacial de la red. En la tercera sección se presenta la aproximación metodológica de los índices de accesibilidad utilizados para cada una de las localidades de la ciudad. La cuarta sección presenta los resultados y el análisis de los indicadores de accesibilidad a la red pública hospitalaria para las diferentes localidades de la ciudad, en donde es posible observar que las más beneficiadas son aquellas con peores condiciones socioeconómicas. La quinta está dedicada a presentar los resultados de satisfacción de los usuarios con la prestación del servicio, así como los indicadores de equidad. De aquí se destaca que, aunque aún existe inequidad en la prestación del servicio de salud, la situación no es tan preocupante como otras ciudades de similar desarrollo. Los comentarios finales se encuentran en la sexta sección.

\section{El nuevo modelo de prestación del servicio de salud en Barranquilla}

El sector salud en Barranquilla, al igual que en la mayoría de ciudades del país, venía atravesando dificultades no solo económicas sino de accesibilidad geográfica, calidad y pertinencia en la prestación de servicios a la comunidad. A partir del gobierno que comenzó en 2008, se inicia una nueva estrategia de prestación de servicios de salud. En el Plan de Desarrollo Oportunidades para Todos, el alcalde Alejandro Char hizo clara la intención de focalizar esfuerzos en ese sentido, a través del planteamiento de una serie de programas y proyectos, que buscaban la reducción de los índices de pobreza y desigualdad, así como el mejoramiento del desarrollo humano (Alcaldía de Barranquilla 2008)².

Con el nuevo esquema de prestación de servicios de salud, se replanteó y reorganizó la red pública hospitalaria, creando lo que hoy se conocen como "Pasos" (Puntos de Atención en Salud Oportunos) y "Caminos" (Centros de Atención Médica Integral Oportunos). Los primeros, son los centros de salud que están pensados para localizarse muy cerca de la comunidad, y cuyo fin es el de prestar un servicio inicial de primera mano, en donde los pacientes son evaluados y, de ser necesario, remitidos a centros más especializados. Los Caminos, por otro lado, están conformados por las instituciones que prestan un servicio de segundo nivel, en donde se atiende a pacientes que requieren tratamientos de mayor complejidad, o en donde se encuentran disponibles servicios de salud especializados. Por ejemplo, solo unos pocos Pasos cuentan con el servicio de urgencias

2 Dentro de los programas del Plan, se proyectaba el avance hacia el aseguramiento universal, el mejoramiento en atención a la población pobre no asegurada, la puesta en marcha de un nuevo esquema de atención básica, y la promoción y prevención de la salud. Adicionalmente, se consideró avanzar en el mejoramiento de la calidad en la prestación de los servicios, así como en el control y vigilancia del sector y sus recursos. 
en sus instalaciones, ya que inicialmente fueron concebidos como puntos de evaluación médica de primera mano.

Los Caminantes de la Salud es otra estrategia sincronizada con la red pública hospitalaria. Son un grupo de profesionales que se encargan de visitar los barrios casa a casa, con el fin de detectar posibles problemas de salud en la comunidad, cumpliendo el papel de promoción y prevención. Esta estrategia lo que busca es incentivar la demanda de los servicios de salud por parte de la población de escasos recursos, recolectando información acerca de las condiciones generales de esa población que ayudarán a focalizar programas de promoción y prevención de enfermedades en comunidades particulares. La articulación de estas tres estrategias, Caminantes, Pasos y Caminos, en coordinación con la red hospitalaria, permiten la rápida y oportuna detección y atención de los principales riesgos de morbilidad que afectan a la población.

A pesar de las medidas adoptadas en el marco de este nuevo modelo, el Distrito detectó falencias de tipo operativo y administrativo por parte del operador que impedía la optimización del recurso humano y económico para el cumplimiento de los objetivos propuestos por el Distrito. Hasta el año 2011, la red prestadora de servicios de salud estaba a cargo de la Caja de Previsión Social de Comunicaciones (CAPRECOM), la cual venía presentando problemas administrativos y de incumplimiento en las metas propuestas e inconvenientes con los sistemas de información. Por esta razón, a partir de enero de 2012 el gobierno local encarga a un nuevo operador, la IPS Universitaria de Antioquia, para que se haga cargo de la red prestadora 3 .

\section{Capacidad instalada, cobertura y prestación de servicios de salud}

Con el fin de mejorar la capacidad instalada y la cobertura en salud, la administración Char a partir de 2008, puso en marcha la transformación y expansión de la red de instituciones de primer y segundo nivel (Pasos y Caminos), respectivamente. Debido a que hasta el momento no se contaba con una conexión espacial entre los distintos niveles de complejidad, el primer peldaño en la implementación de esta novedosa estrategia fue la construcción y puesta en marcha de nuevas IPS de primer y segundo nivel en lugares de baja cobertura.

Para dar una idea de la magnitud del proyecto, durante la Administración Char se construyeron más de veinte nuevas IPS entre Pasos y Caminos, especialmente en las localidades Suroccidente, Suroriente, Metropolitana y Riomar (Gráfico 1). Cepeda (2013) encontró que en las localidades Suroriente y Metropolitana es en donde se localiza la población más vulnerable.

3 Dentro de los avances que se han logrado están la realización de pagos oportunos a los profesionales de la salud, la modernización de los sistemas de información que incluyen la sistematización de las historias clínicas y la conectividad de toda la red hospitalaria, la capacitación del personal médico y administrativo, la modernización de equipos médicos y hospitalarios así como la adecuación de la infraestructura física, el restablecimiento de cirugías ambulatorias, y el incremento en el número de ambulancias, entre los más destacados. 
Gráfico 1. Red pública hospitalaria de Barranquilla

a. 2008

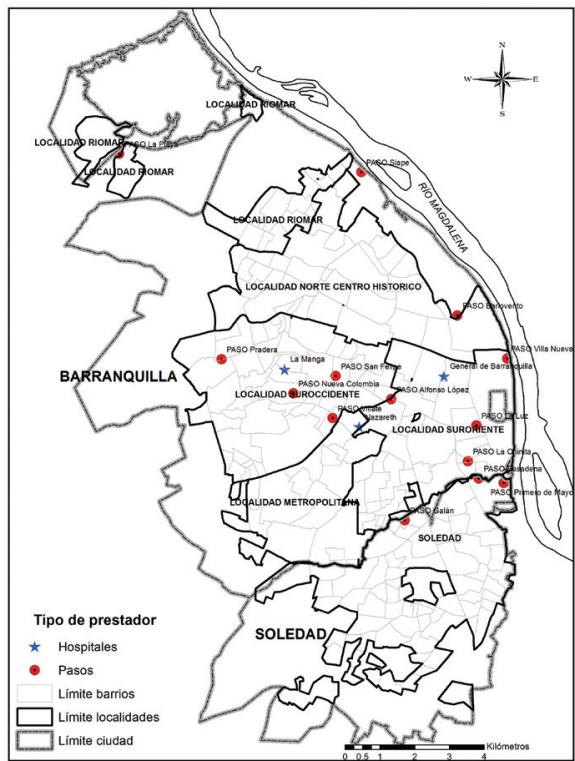

b. 2013

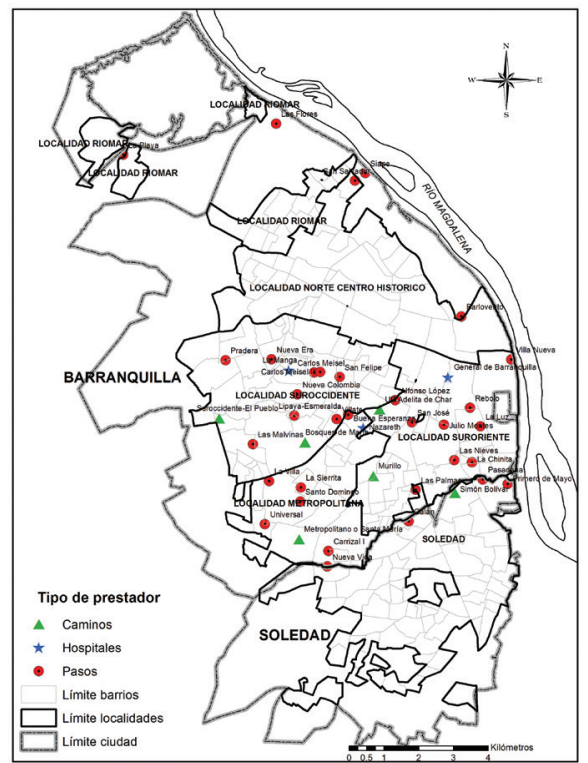

Fuente: elaboración propia con base en información de la IPS Universitaria 2013.

En promedio, la inversión en construcción y puesta en marcha de cada Paso fue de alrededor \$1.500 millones de pesos, dependiendo del tipo de servicios que presta cada uno. Del mismo modo, se llevó a cabo la adecuación y modernización de otros hospitales de la red. Por ejemplo, el Hospital Salud Metropolitana surge de la restructuración de un antiguo puesto de salud que, luego de una inversión cercana a los $\$ 6.500$ millones, quedaría dotado de servicios como urgencias, hospitalización, quirófano, laboratorio y medicina general y especializada, entre otros. El Gráfico 1 muestra la Red Pública Hospitalaria antes y después de la implementación del nuevo esquema. Lo que es evidente es la mayor cobertura espacial de la población barranquillera, lo que debería traducirse en una mayor y mejor disponibilidad de los servicios de salud. La Tabla l presenta las cifras de algunos de los principales indicadores del sector salud como son número de camas, de servicios disponibles y de personal médico en cada una de las localidades, haciendo una comparación entre la situación en 2008 y en 2013. 
Tabla 1. Cambios en la capacidad instalada, servicios y personal médico disponible en la Red Pública Hospitalaria de Barranquilla

\begin{tabular}{|c|c|c|c|c|}
\hline Localidad & Variable & 2008 & 2013 & $\begin{array}{c}\text { Incremento } \\
\text { (\%) }\end{array}$ \\
\hline \multirow{3}{*}{ Metropolitana } & Camas & 24 & 69 & $188 \%$ \\
\hline & Servicios & 24 & 159 & $563 \%$ \\
\hline & Personal médico & 61 & 158 & $159 \%$ \\
\hline \multirow{3}{*}{$\begin{array}{l}\text { Norte - } \\
\text { Centro histórico }\end{array}$} & Camas & 2 & 3 & $50 \%$ \\
\hline & Servicios & 22 & 35 & $59 \%$ \\
\hline & Personal médico & 9 & 14 & $56 \%$ \\
\hline \multirow{3}{*}{ Riomar } & Camas & 1 & 2 & $100 \%$ \\
\hline & Servicios & 14 & 35 & $150 \%$ \\
\hline & Personal médico & 13 & 29 & $123 \%$ \\
\hline \multirow{3}{*}{ Suroccidente } & Camas & 31 & 217 & $600 \%$ \\
\hline & Servicios & 50 & 203 & $306 \%$ \\
\hline & Personal médico & 36 & 298 & $728 \%$ \\
\hline \multirow{3}{*}{ Suroriente } & Camas & 120 & 177 & $48 \%$ \\
\hline & Servicios & 132 & 289 & $119 \%$ \\
\hline & Personal médico & 240 & 419 & $75 \%$ \\
\hline
\end{tabular}

Nota: la información para 2008 y 2013 corresponde al número de camas, al número de servicios disponibles y al número de personal médico en los centros de atención (Pasos (centros de salud), Caminos y Hospitales) de la Red Pública Hospitalaria dentro de cada una de las localidades de Barranquilla. Con el fin de ofrecer una medida de la diferencia entre la oferta y demanda de los servicios de salud en la salud, el Anexo 1 presenta para 2010 los indicadores de déficit de los más importantes servicios prestados por la Red.

Fuente: elaboración propia con base en información de la IPS Universitaria 2013.

Luego de realizar el balance de los servicios disponibles en cada localidad, se encontró que los mayores incrementos se presentaron en las localidades Metropolitana y Suroccidente. En la primera se destaca el aumento del número de servicios de la red pública y en la segunda el aumento del número de camas y el personal médico disponible. El Gráfico 2 muestra la disponibilidad de servicios según el tipo de prestador y su tamaño.

Lo que se puede observar es que las instituciones de las localidades ubicadas al sur de la ciudad (Metropolitana y Suroriente), son las que en la actualidad tienen una mayor dotación de servicios de salud disponibles de toda la red pública. En términos relativos a la población, mientras que entre 2002-2004 el número de camas disponibles por cada 1.000 habitantes en Barranquilla era 1,6, entre 2009-20ll habría aumentado a cerca de 2,8, muy por encima del promedio nacional (1,4), de Cali $(2,1)$, de Medellín (2,1) y de Bogotá (1,7), y muy cerca de las tres camas por cada 1.000 habitantes que tiene, en promedio, un país como los Estados Unidos (Orozco 2013). Estos resultados reflejan no solo avances en términos 
absolutos sino relativos de la capacidad instalada y los servicios prestados por parte de la red pública durante los últimos cinco años

Gráfico 2. Red pública hospitalaria de Barranquilla por servicios disponibles, 2013

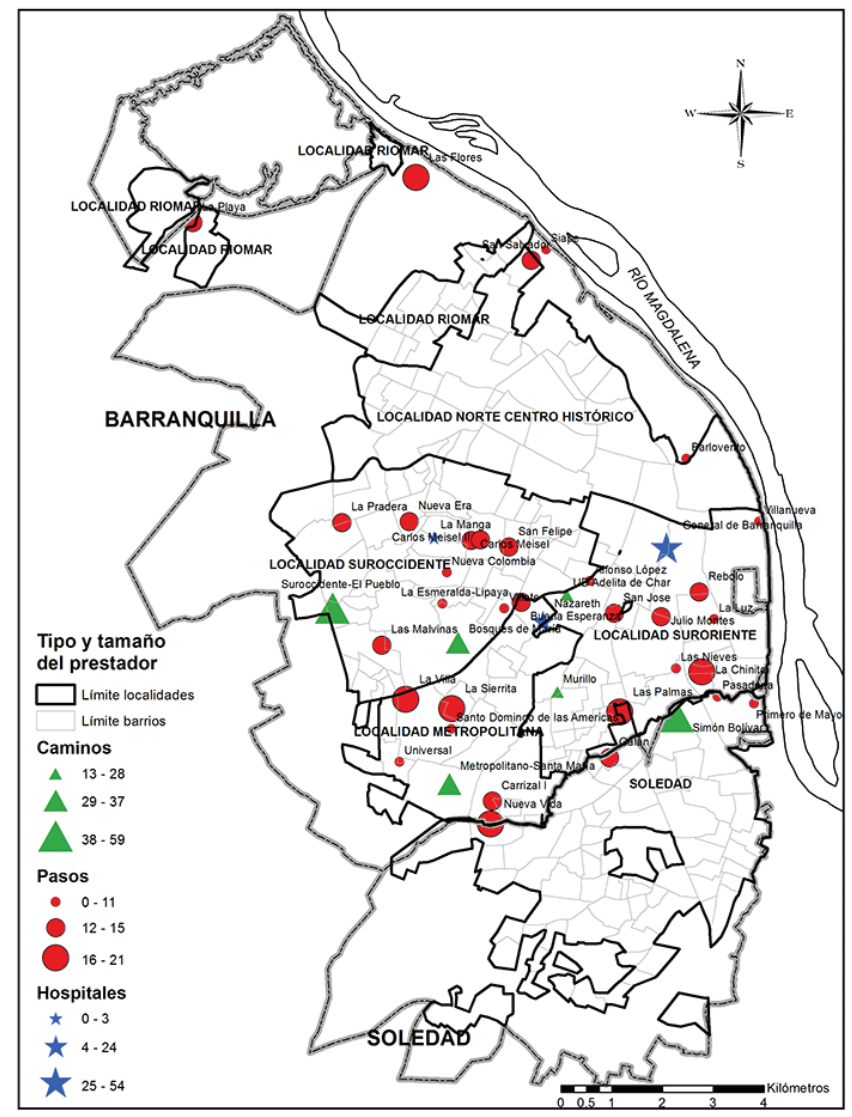

Nota: el tamaño de las figuras representa la disponibilidad del número de servicios que presta cada institución.

Fuente: elaboración propia con base en información de la IPS Universitaria 2013.

\section{Estrategia metodológica}

El objetivo de esta sección es presentar las dos aproximaciones a través de las cuales se pretende dar respuesta a los interrogantes planteados al inicio del documento. El primero es establecer si la nueva red pública hospitalaria de Barranquilla mejoró la accesibilidad geográfica de los habitantes, y el segundo es determinar qué tan equitativo resulta ser el servicio de salud en la ciudad cuando se compara con sus pares de similar nivel de desarrollo.

4 El Anexo 1 presenta una comparación detallada de la capacidad instalada, en términos del número de camas y de estancias, en los centros de la Red Pública Hospitalaria. 


\subsection{Medidas de accesibilidad geográfica}

Para el caso de la accesibilidad geográfica no existe una medida única a través de la cual sea posible aproximar el acceso a servicios en las áreas. Dentro de las medidas más utilizadas están la distancia más cercana, la distancia media a todos los servicios, las medidas de gravedad, y el número de servicios disponibles a cierta distancia o tiempo de recorrido (Higgs 2004). Así, la distancia es un elemento fundamental, y tampoco existe una medida estándar ${ }^{5}$. Sin embargo, Apparicio et al. (2008), demostraron que la distancia cartesiana está fuertemente correlacionada con la distancia más precisa que representa aquella calculada a través de la red vial. El cálculo de las distancias utilizando la red vial es más preciso y ofrece mayores ventajas que el uso de distancias lineales entre dos puntos. Sin embargo, como mencionan Christie y Fone (2003), existen restricciones de información en muchas ciudades que afectarían los cálculos de los tiempos de viaje, como la relativa a congestión vial en cada tramo de la red vial.

Por restricciones de información, en este estudio se utilizará la distancia cartesiana. Luego de determinar las coordenadas geográficas para cada uno de los Pasos, Caminos y Hospitales de la Red Pública, es posible calcular la distancia cartesiana entre cada uno de ellos y un punto de referencia en cada localidad. Debido a que sería arbitrario tomar un solo punto como origen de la distancia (el punto medio de la localidad, por ejemplo), se decidió utilizar tres puntos de referencia: el punto medio de la localidad, el barrio más poblado en cada localidad, y el segundo barrio más poblado de cada una de ellas.

Una segunda aproximación consiste en calcular un indicador de accesibilidad ajustado por la demanda potencial del servicio de salud. Existen varias medidas que intentan aproximar el concepto de accesibilidad a los servicios de salud. La más sencilla es el cálculo de la razón entre la capacidad del prestador y la población, en donde el concepto de capacidad puede ser extendido a indicadores como el número de camas, el número de servicios disponibles por parte del prestador o el personal médico disponible. El concepto espacial es incorporado a través de las áreas geográficas determinadas, tales como municipios, localidades o barrios. Así como la facilidad de cálculo de este indicador es una ventaja, este tiene otras desventajas, relacionadas principalmente con la definición y el tamaño de las áreas geográficas de referencia, lo cual puede llevar a resultados engañosos al no incorporar una medida de la demanda del servicio. Otra medida recientemente utilizada, gracias a la disponibilidad de datos georreferenciados, es la que aproxima la dificultad de acceso a través de la distancia media o mínima a los prestadores de salud. El principal problema de esta medida es que no toma en consideración variables como la capacidad de los prestadores ni el tamaño poblacional.

Una tercera medida, que tiene en cuenta la capacidad instalada de los prestadores y la distancia de los mismos a la población, es la conocida como medida de gravedad. Siguiendo a Unal, Chen y Waldorfd (2007), se define una medida de accesibilidad espacial en la ubicación $k\left(A_{k}\right)$, la cual depende de la capacidad de

5 Algunas de las más utilizadas son la distancia cartesiana, la distancia más corta a través de la red vial, y aquella aproximada como el menor tiempo de recorrido a través de la red vial. 
cada uno de los prestadores del servicio, $S_{\mathrm{j}}$, afectado por una tasa de descuento que captura la dificultad que tiene la población para llegar desde la ubicación $k$ a la ubicación $j, f\left(t_{\mathrm{kj}}\right)^{6}$,

$$
A_{k}=\sum_{j=1}^{J} S_{j} f\left(t_{k j}\right)
$$

Guagliardo (2004) había mencionado ya que uno de los mayores inconvenientes de las medidas de gravedad era que solo tenían en cuenta el lado de la oferta. Para resolver esto, Luo y Wang (2003) proponen incorporar una medida de la variabilidad espacial de la demanda para los servicios ofrecidos por los prestadores, $V_{\mathrm{i}}$, que para el caso de la provisión del servicio de salud, podría ser el número de camas, el número de servicios disponibles y la cantidad de personal médico. En este caso, el factor de demanda se especifica como

$$
V_{j}=\sum_{k=1}^{J} P_{k} f\left(t_{k j}\right)
$$

en donde el nuevo término $P_{\mathrm{k}}$ corresponde a la población como demanda potencial de los servicios de salud, que debe también ser incorporada en el primer término.

Son dos los objetivos para llevar a cabo este ejercicio para el caso particular de Barranquilla. El primero de ellos, es el de obtener una medida de accesibilidad geográfica relativa de la población de cada una de las localidades a la red pública hospitalaria disponible en su misma localidad ${ }^{7}$. Recordemos que la accesibilidad es una condición necesaria determinante en la medición de la calidad del servicio de salud. El segundo objetivo, es observar cómo cambió el ordenamiento en términos de accesibilidad de las localidades luego del cambio en el modelo de prestación del servicio de salud pública en el Distrito a partir de 2008. Para el caso particular de Barranquilla, el indicador para la localidad $i$ toma la siguiente forma:

$$
A_{i}=\sum_{j=1}^{n} \frac{\sum_{k=1}^{3} P_{i(k)} S_{j} d_{i(k) j}^{-\beta}}{\sum_{i=1}^{5} \sum_{k=1}^{3} P_{i(k)} d_{i(k) j}^{-\beta}}
$$

en donde el indicador de accesibilidad $A i$ está determinado por la población $P$ en cada uno de los tres posibles puntos de origen de los pacientes (punto medio de la localidad, el barrio más poblado y el segundo barrio más poblado). $S_{\mathrm{j}}$ representa la capacidad instalada de los prestadores (número de camas por ejemplo), y $d$ es la distancia lineal entre cada uno de los tres puntos posible de origen de

6 Como mencionan los autores, el término $f\left(t_{k j}\right)$ se aproxima frecuentemente a una función inversa de la distancia entre $k$ y $j, t_{k j}^{-\beta}$, en donde $\beta$ representa el factor de decaimiento, que por abarcar múltiples factores (atributos de los usuarios que recorren las distancias, y factores demográficos y socioeconómicos) son definidos en forma subjetiva. Para evitar dichas dificultades y por simplicidad se asumirá en este caso que $\beta=1$.

7 Debe tenerse en cuenta que para el caso del presente documento el concepto de accesibilidad que se quiere aproximar es el potencial calculado en función de variables geográficas, demográficas y de capacidad instalada de la red de prestadores de salud. 
la población, $i_{(\mathrm{k})}$, a cada uno de los prestadores del servicio de salud $j$ (en este caso Pasos, Caminos y Hospitales). $\beta$ representa el coeficiente de decaimiento de gravedad, que en algunos caso se denomina coeficiente de fricción de viaje (Guagliardo 2004, 5). Debido a que el objetivo es obtener una medida relativa de accesibilidad a través de las localidades, y debito también a que para el resultado de los índices absolutos no hay una unidad estándar para su interpretación, estos fueron re-escalados para que tomen valores entre 0 y 1 , en donde o representa la peor accesibilidad y 1 la mejor.

\subsection{Equidad en la prestación de los servicios de salud}

En cuanto a la intención de aproximar, en alguna medida, el grado de equidad con el que se está prestando el servicio de salud en Barranquilla, se utilizó la información de las encuestas de percepción de los proyectos Cómo vamos en algunas de las principales ciudades. En particular se empleó, a nivel de individuos, el grado de satisfacción reportado por cada persona ante la pregunta ¿qué tan insatisfecho o satisfecho está usted con el servicio de salud que ha recibido? Debido a las características particulares de la variable dependiente, en este caso el nivel de satisfacción con el servicio de salud, se utiliza un Modelo de Umbral Generalizado, que pertenece a la familia de Modelos de Respuesta Ordenada. Estos modelos representan una mejora metodológica sobre los modelos de respuesta binaria (logit y probit), al considerar las múltiples categorías de respuesta, pero además tienen ventaja sobre los modelos multinomiales ya que a las diferentes opciones de respuesta les corresponde un ordenamiento específico. Estas características permiten que haya un conjunto particular de parámetros para cada umbral, lo cual implica que los parámetros varíen a lo largo de cada una de las opciones de respuesta.

$$
P(Y=j \mid X ; \theta)=F\left(k_{j}-X^{\prime} \beta_{j}\right)-F\left(k_{j-1}-X^{\prime} \beta_{j-1}\right)
$$

en donde $Y$ corresponde a la variable dependiente que toma valores entre $j=1, \ldots$, $J$, que en este caso es el nivel de satisfacción; $X$ es el conjunto de variables explicativas de la satisfacción; $F($.) representa la función de densidad acumulada que depende de los valores del umbral, $k_{\mathrm{j}}$, y de las variables explicativas y sus correspondientes coeficientes, $X^{\prime} \beta_{\mathrm{i}}$. Es importante observar que en este caso, contrario a lo que ocurre con los modelos de respuesta ordenada tradicionales, el conjunto de parámetros contenidos en $\beta$ varían a lo largo de la distribución con respecto a los valores del umbral, $j$. Esto permite calcular estas variaciones para cada una de las categorías de satisfacción.

\section{Accesibilidad geográfica a los servicios de salud}

Esta sección presenta un análisis de accesibilidad a los servicios de la red pública hospitalaria, a través de la comparación entre la situación antes y después de la implementación del nuevo modelo de prestación del servicio. Según Unal, Chen y Waldorfd (2007), la accesibilidad es una condición necesaria pero 
no suficiente para garantizar la calidad y la igualdad en la prestación de los servicios de salud.

Es importante mencionar que los términos "acceso" y "accesibilidad" han tenido variadas connotaciones en la literatura, las cuales han surgido, principalmente, en el contexto de la provisión de servicios de salud. Por ejemplo, para Penchasky y Thomas (1981), el término acceso está relacionado con la posibilidad o disposición de los usuarios de recibir el servicio, es decir, depende del grado de ajuste entre el usuario y la red pública hospitalaria. Por otro lado, Gulliford et al. (2002) van más allá, y tienen en consideración las demás barreras, aparte de las geográficas, tales como las económicas, institucionales y culturales. Los autores definen dos términos, "tener acceso" y "conseguir acceso", en donde el primero lo relacionan con el lado de la oferta (disponibilidad del servicio), y el segundo con las restricciones (económicas, institucionales y culturales) que enfrentan los individuos para obtener el servicio. Para el caso particular de este documento, el término acceso estará relacionado con las barreras geográficas, la distancia en particular.

Recordemos que las largas distancias que deben recorrer los usuarios hasta los puntos de atención en salud es uno de los principales obstáculos al acceso de servicios médicos de calidad. Penchansky y Thomas (1981) mencionan los siguientes aspectos como algunos de los más importantes obstáculos que pueden afectar la adecuada prestación de los servicios de salud: la asequibilidad (en términos económicos), la capacidad instalada (disponibilidad de camas), la disponibilidad (de los servicios adecuados), y la accesibilidad (cercanía o fácil movilización hacia los prestadores del servicio).

Comber, Brundsdon y Radburn (2011) mostraron que son tres los principales factores que influyen en la percepción de acceso a los servicios de salud: el estado de salud, tener vehículo y la distancia a la red hospitalaria. Este último factor, para el caso del Reino Unido, resultó significativo solamente en cuanto al acceso a los centros de salud prioritaria, pero no a los de mayor complejidad (hospitales). La Tabla 2 muestra los resultados de un primer ejercicio, el cual consiste en calcular la distancia a la que se encuentra la población de los prestadores de salud. Aquí se presentan no solamente los cálculos de las distancias actuales, sino una comparación antes de los cambios en el esquema de salud, y la ganancia o pérdida en términos de distancia ${ }^{8}$.

8 El Anexo 2 presenta la distribución de la distancia promedio general de la población a la red Pública Hospitalaria antes y después de la implementación del nuevo modelo de prestación del servicio de salud. 
Tabla 2. Distancia entre la población y la red pública hospitalaria (Pasos, Caminos y hospitales) de Barranquilla

\begin{tabular}{|c|c|c|c|c|}
\hline \multirow{2}{*}{ Localidad } & \multirow{2}{*}{ Origen de la distancia } & \multicolumn{2}{|c|}{ Distancias (en mts) } & \multirow{2}{*}{$\begin{array}{c}\text { Diferencia } \\
\text { 2008-2013 (en } \\
\text { mts) }\end{array}$} \\
\hline & & 2008 & 2013 & \\
\hline \multirow{4}{*}{ Metropolitana } & Centro de la localidad & 2.581 & 1.467 & 1.114 \\
\hline & Barrio más poblado & 3.248 & 2.016 & 1.232 \\
\hline & Segundo barrio más poblado & 3.067 & 1.545 & 1.522 \\
\hline & Promedio general & 2.965 & 1.676 & 1.289 \\
\hline \multirow{4}{*}{$\begin{array}{l}\text { Norte - } \\
\text { Centro histórico }\end{array}$} & Centro de la localidad & 3.193 & 3.160 & 33 \\
\hline & Barrio más poblado & 3.157 & 3.320 & -164 \\
\hline & Segundo barrio más poblado & 2.621 & 3.084 & -464 \\
\hline & Promedio general & 2.990 & 3.188 & -198 \\
\hline \multirow{4}{*}{ Riomar } & Centro de la localidad & 2.904 & 2.663 & 241 \\
\hline & Barrio más poblado & 730 & 2.884 & -2.154 \\
\hline & Segundo barrio más poblado & 5.456 & 3.769 & 1.687 \\
\hline & Promedio general & 3.030 & 3.105 & -75 \\
\hline \multirow{4}{*}{ Suroccidente } & Centro de la localidad & 1.239 & 1.412 & -173 \\
\hline & Barrio más poblado & 2.094 & 1.891 & 203 \\
\hline & Segundo barrio más poblado & 1.351 & 1.572 & -221 \\
\hline & Promedio general & 1.561 & 1.625 & -64 \\
\hline \multirow{4}{*}{ Suroriente } & Centro de la localidad & 1.950 & 1.642 & 308 \\
\hline & Barrio más poblado & 1.983 & 1.631 & 352 \\
\hline & Segundo barrio más poblado & 2.109 & 2.082 & 27 \\
\hline & Promedio general & 2.014 & 1.785 & 229 \\
\hline
\end{tabular}

Nota: las distancias fueron calculadas como distancias lineales entre la población (centro de la localidad, barrio más poblado y segundo barrio más poblado) y la red pública hospitalaria (centros de salud y hospitales antes de 2008, y Pasos, Caminos y hospitales en 2013).

Fuente: elaboración propia con base en información cartográfica del IGAC.

Lo que se puede observar es que en dos de las cinco localidades hubo una reducción neta de la distancia que deben recorrer los habitantes para tener acceso al servicio de salud. Para el caso de la Localidad Metropolitana, la distancia se redujo en al menos $1,1 \mathrm{~km}$ cuando se toma como referencia el centro de la localidad, y en $1,2 \mathrm{~km}$ cuando se considera el promedio desde cualquiera de los tres puntos de referencia. Para este último caso, la distancia media a un punto de atención médica pasó de $3 \mathrm{~km}$ en 2008 a 1,6 en la actualidad. La otra localidad con reducciones netas es la Localidad Suroriente, para la cual la distancia promedio desde cualquier punto de referencia se redujo de $2 \mathrm{~km}$ en 2008 a 1,7 km en 2013. La mayor reducción se presentó para quienes habitan el barrio más poblado.

Para las tres localidades restantes, los resultados son diferenciales dependiendo del punto de referencia de la población. Por ejemplo, para la Localidad Norte-Centro histórico la distancia se redujo levemente solo para el caso en el 
que se toma el centro de la localidad como punto de referencia de la población. Para los otros dos casos (barrio más poblado y segundo barrio más poblado), las distancias a los puntos de atención pública en salud aumentan levemente entre 164 y 464 metros. Algo similar ocurre en el caso de la Localidad Suroccidente, aunque en este la disminución en la distancia se produce para los habitantes del barrio más poblado. En la Localidad Riomar, la expansión de la red pública hospitalaria trajo consigo reducciones en las distancias para aquellos que habitan cerca del centro de la localidad $(0,241 \mathrm{~km})$ y en el segundo barrio más poblado $(1,6 \mathrm{~km})$. Sin embargo, para el caso de quienes habitan en el barrio más poblado, la distancia aumentó en $2,1 \mathrm{~km}$. La razón muy sencilla es que el único centro de salud que existía antes de 2008 estaba localizado más cerca (a 0,7 km) del barrio más poblado (La Playa) y bastante lejos del segundo barrio más poblado (a 5,4 $\mathrm{km}$ ), por lo que la apertura del Paso Las Flores al nororiente de la localidad aumentaba la distancia desde el barrio más poblado pero reducía la del segundo barrio más poblado. Bajo estas consideraciones, la estrategia de apertura del nuevo Paso Las Flores da la oportunidad a los habitantes del segundo barrio más poblado de tener acceso fácil a la prestación del servicio de salud. Antes de 2008 los habitantes de este barrio debían recorrer 5,4 km al centro de salud más cercano en su localidad, y en la actualidad deben recorrer solamente $2 \mathrm{~km}$ para tener acceso al servicio de salud?.

Cuando se calcula el índice de accesibilidad, la Tabla 3 presenta los resultados utilizando tres medidas diferentes de capacidad: camas, servicios médicos y personal médico. Las dos primeras columnas muestran una comparación de los índices para cada localidad entre 2008 y 2013, mientras que las columnas 3 y 4 presentan una comparación para el ranking de accesibilidad, es decir, el cambio relativo en términos de accesibilidad a los servicios distritales de salud que se presentaron luego del cambio del modelo en la ciudad. Es importante mencionar que para el caso del acceso a las camas disponibles no se esperan grandes cambios entre 2008 y 2013. La razón es que a pesar del importante aumento en el número de instituciones prestadoras, la mayoría corresponden a Puntos de Atención en Salud Oportunos (Pasos) que, por su naturaleza de ser puntos de atención básica de primera mano, no cuentan con disponibilidad de camas.

Los resultados para el número de camas disponibles muestran que solo hubo cambios en dos de las cinco localidades que forman el Distrito. La Localidad Metropolitana, que antes de 2008 ocupaba el primer lugar en cuanto a accesibilidad de camas, pasó a ocupar el tercer lugar, mientras que la Localidad Suroccidente ganó dos posiciones ocupando el primer lugar en la actualidad. Las demás localidades no presentaron cambios relativos en su ordenamiento.

9 Es importante mencionar que bajo este análisis se está calculando la accesibilidad de los habitantes a los puntos de atención de salud pública dentro de su propia localidad. En algunos casos, como en la localidad de Riomar por ejemplo, algunos habitantes se encuentran ubicados más cerca de puntos de atención de la Localidad Norte-Centro histórico. 
Tabla 3. Índice relativo de accesibilidad a los servicios de la Red Pública Hospitalaria de Barranquilla

\begin{tabular}{|l|c|c|c|c|}
\hline \multirow{2}{*}{ Localidad } & \multicolumn{2}{c|}{$\begin{array}{c}\text { a. Camas } \\
\text { Indice de accesibilidad } \\
\text { relativo (ARi) }\end{array}$} & \multicolumn{2}{c|}{ Ranking } \\
\cline { 2 - 5 } & 2008 & 2013 & 2008 & 2013 \\
\hline Metropolitana & 1,00 & 0,44 & 1 & 3 \\
\hline Norte-Centro histórico & 0,00 & 0,00 & 4 & 4 \\
\hline Riomar & 0,00 & 0,00 & 5 & 5 \\
\hline Suroccidente & 0,33 & 1,00 & 3 & 1 \\
\hline Suroriente & 0,76 & 0,75 & 2 & 2 \\
\hline
\end{tabular}

\begin{tabular}{|l|c|c|c|c|}
\hline \multirow{2}{*}{\multicolumn{1}{c}{ Localidad }} & \multicolumn{1}{c|}{$\begin{array}{c}\text { Indice de accesibilidad } \\
\text { relativo (ARi) }\end{array}$} & \multicolumn{2}{c|}{ Ranking } \\
\cline { 2 - 5 } & 2008 & 2013 & 2008 & 2013 \\
\hline Metropolitana & 1,00 & 0,78 & 1 & 3 \\
\hline Norte-Centro histórico & 0,12 & 0,00 & 4 & 5 \\
\hline Riomar & 0,33 & 0,86 & 3 & 2 \\
\hline Suroccidente & 0,00 & 0,30 & 5 & 4 \\
\hline Suroriente & 0,61 & 1,00 & 2 & 1 \\
\hline
\end{tabular}

\begin{tabular}{|l|c|c|c|c|}
\hline \multirow{2}{*}{\multicolumn{1}{|c|}{ Localidad }} & \multicolumn{1}{c|}{$\begin{array}{c}\text { Indice de accesibilidad } \\
\text { ajustado (ARi) }\end{array}$} & \multicolumn{2}{c|}{ Ranking } \\
\cline { 2 - 5 } & 2008 & 2013 & 2008 & 2013 \\
\hline Metropolitana & 1,00 & 0,52 & 1 & 4 \\
\hline Norte-Centro histórico & 0,00 & 0,00 & 5 & 5 \\
\hline Riomar & 0,15 & 0,55 & 3 & 3 \\
\hline Suroccidente & 0,07 & 0,77 & 4 & 2 \\
\hline Suroriente & 0,57 & 1,00 & 2 & 1 \\
\hline
\end{tabular}

Fuente: elaboración propia con base en información cartográfica del IGAC, y de capacidad instalada de los prestadores de la IPS Universitaria de Barranquilla, 2008 y 2013.

En cuanto a las otras dos medidas de capacidad de los prestadores de la red pública, es de esperar mayores cambios debido a que cada nueva institución implica el aumento en el número de servicios prestados por la red pública en la localidad correspondiente, así como el aumento en la cantidad de personal médico. La Localidad Metropolitana que antes de 2008 ocupaba el primer lugar en cuanto a servicios disponibles y personal médico, esto a pesar de contar con una sola institución prestadora (el Hospital Nazareth tenía la segunda mayor oferta de servicios y personal médico en la ciudad), pasa a ocupar el tercer y cuarto lugar en servicios y personal médico, respectivamente. Esto implica un 
cambio significativo en términos de la descentralización del servicio de salud en la localidad y comparativamente en el Distrito. Las localidades ganadoras en términos de acceso a los servicios de salud, es decir, aquellas que mejoraron frente a las demás con el nuevo sistema fueron Riomar, Suroccidente y Suroriente en términos de los servicios disponibles y, Suroccidente y Suroriente en términos del personal médico disponible.

Hasta el momento ha quedado claro que el nuevo esquema de prestación de servicios de salud en el Distrito mejoró la accesibilidad por parte de la población dentro de cada una de sus localidades. Sin embargo, debido a que no existe restricción de atención por localidades, es decir, que los pacientes pueden acudir a cualquier centro de atención público así no se encuentre localizado dentro de su localidad, queda la pregunta de si a nivel del Distrito como un todo mejoró la accesibilidad de la población al servicio de salud ${ }^{10}$.

Para responder a esta pregunta, en el Gráfico 3 se presenta la relación entre el porcentaje acumulado de la población y la distancia que deben recorrer los barranquilleros al punto de atención más cercano, para 2008 y para 2013. El panel (a) muestra el caso frente al acceso de los puntos de atención básica (Pasos), y el panel (b) tiene en cuenta el acceso de la población a centros de atención más especializados (Caminos y Hospitales). Las distancias se calcularon desde el punto central de cada barrio hasta el punto de atención en salud más cercano. RoseroBixby (2004) utilizaron esta aproximación para establecer el nivel de acceso a los servicios de salud en Costa Rica.

Gráfico 3. Proporción de la población acumulada según la distancia más corta a los servicios de la red pública hospitalaria en Barranquilla

a. Pasos (centros de salud)

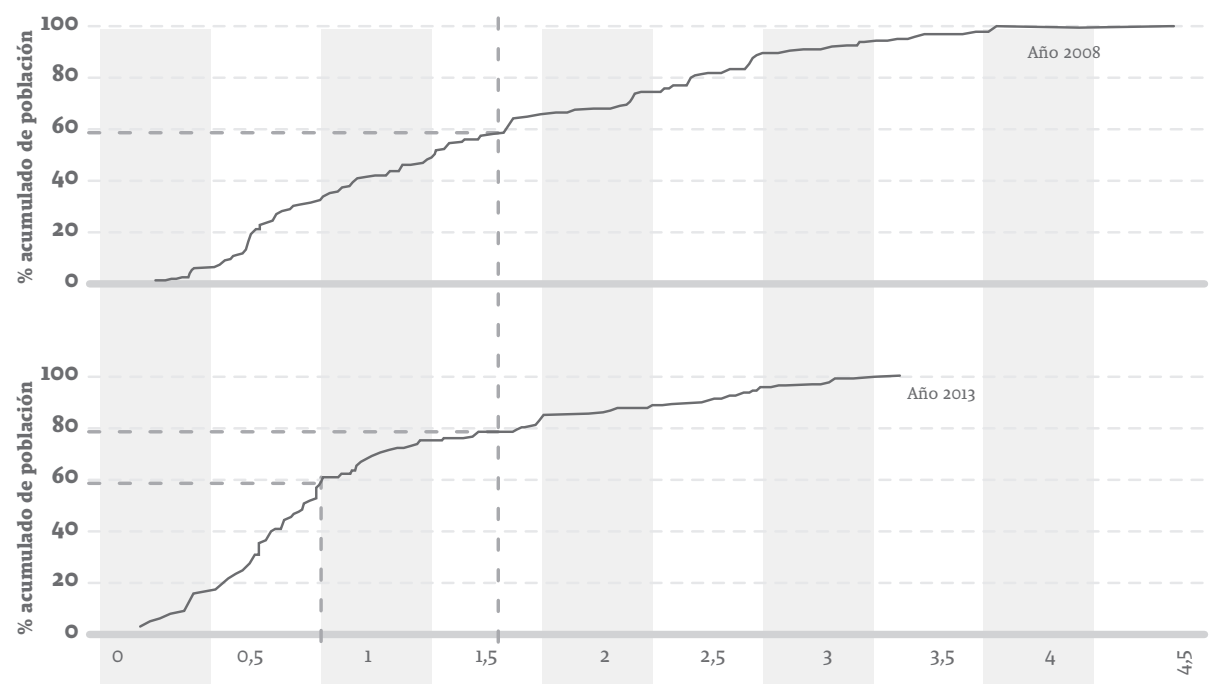

Distancia $(\mathrm{km})$ con el fin de establecer la accesibilidad de la población mayor de 65 años a los servicios de salud. 
b. Caminos y hospitales

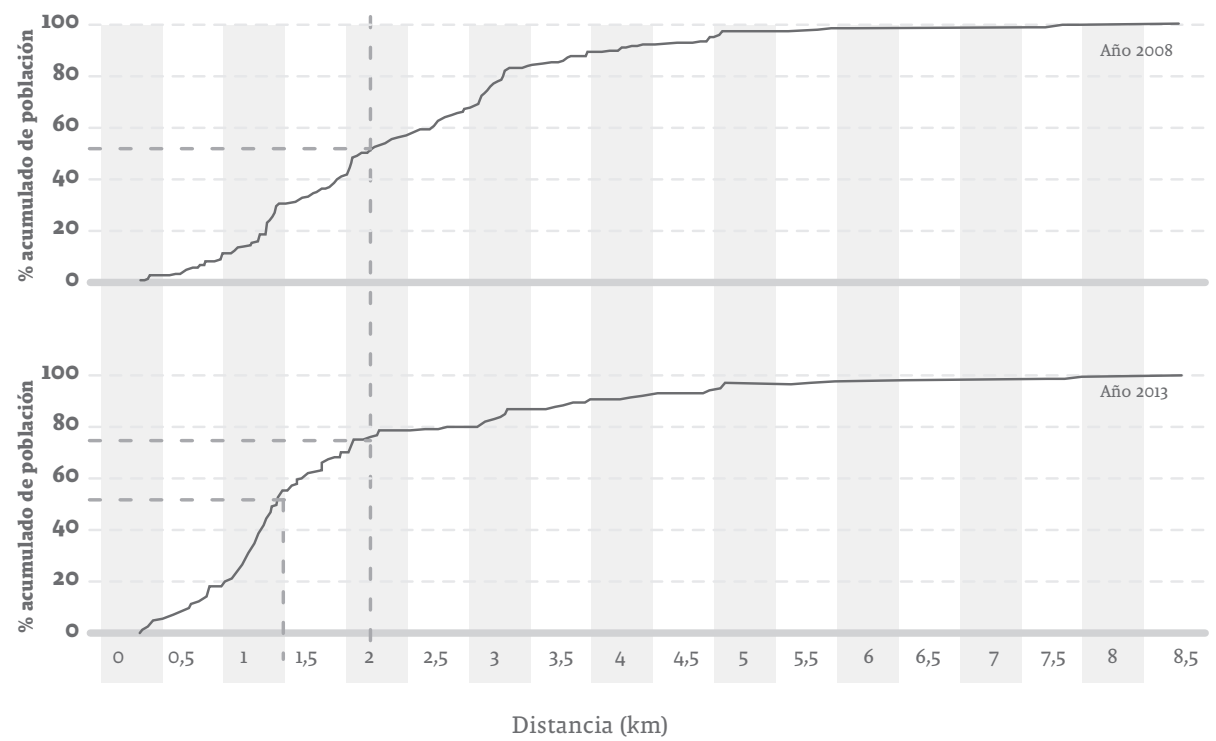

Nota: los porcentajes acumulados de población y distancia mínima se realizaron para el conjunto de barrios del Distrito.

Fuente: elaboración propia con base en información cartográfica del IGAC, y de capacidad instalada de los prestadores de la IPS Universitaria de Barranquilla, 2008 y 2013.

Este ejercicio tiene la utilidad de presentar simultáneamente un indicador de cambio en la distancia mínima recorrida para llegar a un punto de atención, y el cambio de la relación conjunta entre la población y la distancia mínima recorrida. Si se observa el panel (a), se puede ver que la proporción de la población acumulada con respecto a la distancia mínima se contrajo en 2013. Por ejemplo, mientras que en 2008 tan solo el $60 \%$ de la población se encontraba a una distancia mínima de 1,6 km del centro de salud (Pasos) más cercano, en 2013 la proporción de la población que se encuentra a esta distancia aumentó al 80\%. Otra forma de leer estos resultados es que mientras en 2008 el $60 \%$ de la población se encontraba a 1,6 km de distancia del punto de servicio más cercano, en 2013 la misma proporción de la población se encuentra a menos de $1 \mathrm{~km}$.

Un resultado similar al anterior se encontró para la distancia mínima que una persona debe recorrer hasta el Hospital o Camino más cercano (panel b). En este caso se puede observar que mientras que en 2008 la mitad de la población se encontraba a $2 \mathrm{~km}$ de distancia de estos centros de atención, en 2013 el 80\% de la población está a esa misma distancia. Dicho de otro modo, mientras que en 2008 la mitad de la población estaba a $2 \mathrm{~km}$ del Hospital o Camino más cercano, en 2013 esa misma población se encuentra a tan solo 1,2 km de distancia, lo cual representa una ganancia sustancial en términos de accesibilidad. Doriwala y Shah (2010) mencionan que la relación en forma de U entre el uso y la distancia de instalaciones proveedoras de servicios comunitarios (como educación, salud o recreación), podría ser explicada por la zonificación espacial de los estratos socioeconómicos. 


\section{Percepción ciudadana}

Una forma indirecta adicional de medir el desempeño en términos de calidad de cualquier servicio son las encuestas de percepción a los usuarios. A través de ellas se captura la satisfacción de estos con respecto al servicio que se les presta y, en algunos casos, las razones por las cuales tiene esa opinión en particular ${ }^{11}$. En Colombia, el ejemplo más representativo de este esquema son las encuestas de percepción que realizan los proyectos Cómo vamos en las principales ciudades del país ${ }^{12}$. Los resultados de estas encuestas se han convertido en una herramienta de seguimiento a las políticas públicas de los gobiernos locales relacionadas con la calidad de vida en las ciudades. Esta sección tiene dos objetivos principales. El primero es el de establecer qué tan satisfechos están los ciudadanos actualmente con el servicio de salud y cómo ha evolucionado dicha percepción durante los últimos años. El segundo objetivo es el de aproximar una medida de equidad en la prestación de los servicios de salud en Barranquilla, determinando si la satisfacción depende en alguna medida de factores socioeconómicos.

El Gráfico 4 resume la opinión general de la población acerca de la prestación del servicio de salud en algunas de las principales ciudades. Para el caso de Barranquilla, hay dos aspectos para resaltar. El primero es una leve disminución en el tiempo de aquellos que se sienten satisfechos con la prestación del servicio de salud; mientras que en 2008 el $67 \%$ de los entrevistados se sentía satisfecho, en 2011 este porcentaje se redujo al 61,5\%. Sin embargo, cuando se observan los resultados para las demás ciudades en todas ellas los niveles de satisfacción son menores en 2011 que en 2008. El segundo resultado, y tal vez más importante es que, a pesar del descenso, en 2011 Barranquilla era la ciudad con los más altos niveles de satisfacción con el servicio de salud, por encima incluso de ciudades como Medellín y Bogotá.

Para verificar si este es un hecho aislado o resultado de una pregunta muy general acerca del tema de la salud, se realizó el mismo ejercicio teniendo en cuenta preguntas diversas relacionadas con el sector de la salud, y los resultados en su mayoría son consistentes, con leves disminuciones en el tiempo para todas las ciudades, y Barranquilla en los primeros lugares.

11 Es importante mencionar que la percepción de satisfacción de los individuos puede ser afectada no solo por la opinión general que tiene el individuo acerca del servicio que recibe, sino por aspectos particulares aislados que haya enfrentado o que esté enfrentando el usuario, y que no necesariamente tiene que ver directamente con el servicio por el que está siendo consultado.

12 Esta iniciativa de carácter privado, que inició en 1998 con Bogotá y que ha sido replicada nacional e internacionalmente, se encarga de hacer un seguimiento sistemático a los principales indicadores de calidad de vida en las ciudades. Actualmente, la Red de ciudades Cómo vamos en Colombia está conformada por Bogotá, Cali, Cartagena, Medellín, Barranquilla, Bucaramanga, Manizales, Valledupar, Pereira e Ibagué. 
Gráfico 4. Percepción de satisfacción con el servicio de salud recibido

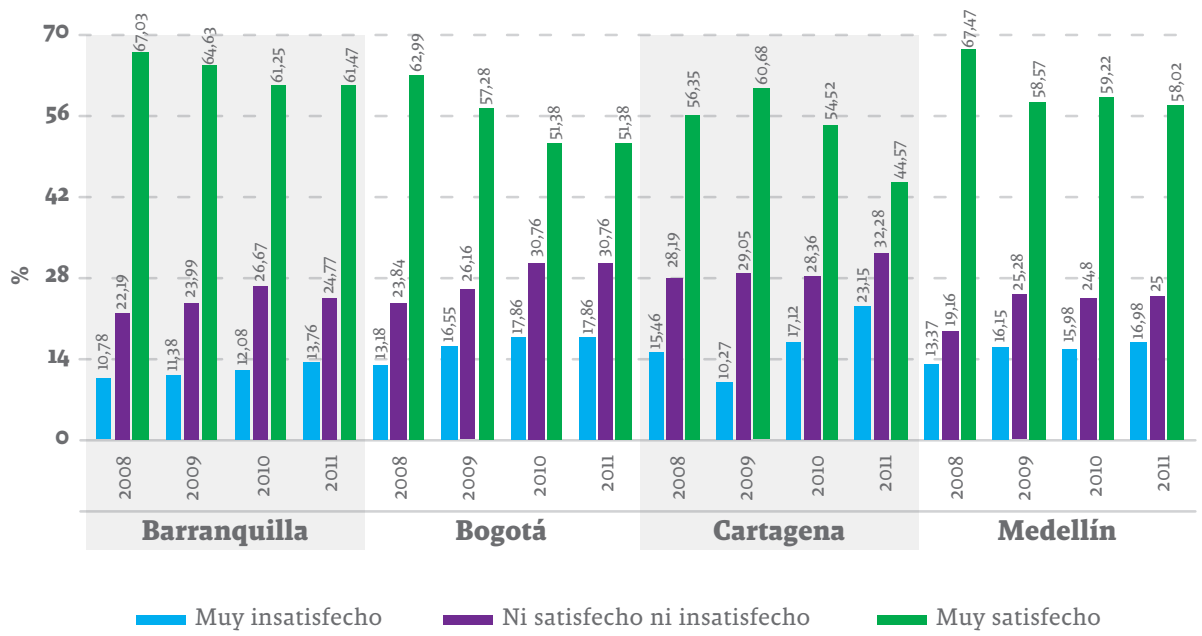

Nota: por conveniencia para la lectura de los resultados, las respuestas fueron reclasificadas en tres grupos: Muy satisfecho (Satisfecho y Muy satisfecho), Ni satisfecho ni insatisfecho, y Muy insatisfecho (Insatisfecho y Muy insatisfecho). La pregunta en particular hecha a los ciudadanos fue: ¿qué tan insatisfecho o satisfecho está usted con el servicio de salud que ha recibido?

Fuente: elaboración propia con base en las encuestas Cómo vamos de las respectivas ciudades, 2008 a 2011.

Por ejemplo, cuando a las personas se les preguntó acerca de la imagen de la Secretaría de Salud del Distrito, a 2011 el 62\% de los barranquilleros manifestaron tener una imagen favorable de esta institución, comparado con Bogotá (41\%), Cartagena (53\%) y Medellín (34\%). Adicionalmente, ante la pregunta de si los entrevistados sienten que en la ciudad se les está garantizando el derecho a la salud, Barranquilla en 2011 era la de más alta percepción con un $65 \%$, comparado con Cartagena (52\%) y Medellín (60\%). Así como lo menciona Orozco (2013), los resultados de las encuestas muestran que las razones más frecuentas por las que los individuos sienten algún grado de insatisfacción con la prestación del servicio de salud son las demoras en la asignación de citas médicas y autorización de procedimientos.

Finalmente, el último ejercicio quiere responder a la pregunta de si los factores socioeconómicos afectan la percepción que tienen las personas acerca de la calidad en la prestación del servicio de salud. Esta es una pregunta fundamental para los formuladores de políticas públicas, ya que es posible establecer algún grado de inequidad en la prestación del servicio. Idealmente, la probabilidad de que una persona se sienta satisfecha o insatisfecha no debería depender de su situación socioeconómica, especialmente cuando el hecho de ser pobre aumenta la probabilidad de sentirse insatisfecho con el servicio recibido.

La Tabla 4 presenta los efectos marginales de los niveles socioeconómicos sobre la satisfacción con el servicio de salud obtenidos de las estimaciones del 
modelo de umbral generalizado ${ }^{13}$. Los resultados están mostrando dos aspectos importantes; el primero es la significancia de los parámetros, es decir, que el hecho de percibirse como una persona pobre es un factor que parece estar afectando el nivel de satisfacción con el servicio de salud.

Tabla 4. Efectos marginales de la percepción de pobreza sobre la satisfacción con la prestación de los servicios de salud en Barranquilla, 2010

\begin{tabular}{|c|c|c|c|c|c|}
\hline \multirow[b]{2}{*}{ Variable dependiente } & \multicolumn{5}{|c|}{2010} \\
\hline & $\begin{array}{c}\text { Muy } \\
\text { insatisfecho }\end{array}$ & $\begin{array}{c}\text { Algo } \\
\text { insatisfecho }\end{array}$ & $\begin{array}{c}\mathrm{Ni} \\
\text { satisfecho } \\
\text { ni } \\
\text { insatisfecho }\end{array}$ & $\begin{array}{c}\text { Algo } \\
\text { satisfecho }\end{array}$ & $\begin{array}{c}\text { Muy } \\
\text { satisfecho }\end{array}$ \\
\hline Percepción de pobreza & $0,023^{* * *}$ & $0,019^{* * *}$ & $0,043^{* * *}$ & $-0,022^{* *}$ & $-0,063^{* * *}$ \\
\hline (Pobre=1) & $(0,009)$ & $(0,007)$ & $(0,015)$ & $(0,009)$ & $(0,022)$ \\
\hline Log-likelihood & & & $-1174,92$ & & \\
\hline Observaciones & & & 840 & & \\
\hline
\end{tabular}

Nota: los resultados provienen de un modelo cuya variable dependiente es el nivel de satisfacción de los individuos con respecto al servicio de salud, y la variable independiente es la percepción de pobreza de los entrevistados (pobre $=1$ ). Como variables de control se incluyeron variables dummy para los grupos de edad y de género. Desviaciones estándar robustas en paréntesis.

Fuente: elaboración propia con base en información de las encuestas Cómo vamos, 2010.

El segundo aspecto tiene que ver más directamente con la dirección de los efectos marginales. Es posible notar que para aquellos que se perciben como pobres aumenta la probabilidad de sentirse insatisfechos o indiferentes con el servicio de salud, a la vez que se reduce la probabilidad de sentirse satisfechos, todo esto con respecto a las personas que no se consideran pobres. Para un caso en particular, los resultados muestran que la probabilidad de estar muy insatisfecho con el servicio de salud aumenta en 0,023 puntos porcentuales (pp) para aquellos que se perciben como pobres con respecto a quienes no se consideran en esta situación ${ }^{14}$.

Los resultados mostraron claramente un efecto de la percepción de pobreza sobre los niveles de satisfacción. Sin embargo, recordemos que esta medida podría estar sobrestimada por el hecho de ser un indicador subjetivo del nivel socioeconómico del individuo. Con el fin de corroborar los resultados anteriores, pero esta vez utilizando una medida estandarizada para categorizar el nivel socioeconómico de las personas, se calcularon los efectos marginales del estrato socioeconómico sobre la satisfacción con el servicio de salud recibido ${ }^{15}$.

13 Estos resultados son robustos cuando las categorías de satisfacción se reagrupan en tres categorías: muy o algo insatisfecho=0,037*; ni satisfecho ni insatisfecho=0,038**; algo o muy satisfecho=-0,075**.

14 Se llevó a cabo el mismo ejercicio pero esta vez utilizando como variable explicativa una variable dummy que indicaba si la persona estaba o no atravesando por una situación económica difícil al momento de la entrevista. Los resultados son muy similares a los de la percepción de la pobreza en términos de significancia, dirección y magnitud de los efectos marginales.

15 Por restricciones de información, esta vez se utiliza la información para 2011 y categorizada en solo tres niveles de satisfacción. 
La Tabla 5 muestra resultados igualmente significativos y con la misma dirección según los niveles de satisfacción. Sin embargo, es posible observar que los efectos son significativamente mayores comparados con los de la percepción de pobreza. Para todo los estratos (comparados con las personas de estrato 6), la probabilidad de sentirse insatisfecho aumenta en cerca de 0,22 pp, a la vez que la probabilidad de sentirse satisfecho disminuye para las personas de todos los estratos con respecto a los individuos de estrato 6 (grupo de referencia).

Tabla 5. Efectos marginales de la condición socioeconómica sobre la satisfacción con la prestación de los servicios de salud en Barranquilla, 2011

\begin{tabular}{|c|c|c|c|}
\hline \multirow[b]{2}{*}{ Variable dependiente } & \multicolumn{3}{|c|}{2011} \\
\hline & $\begin{array}{l}\text { Muy insatisfecho o } \\
\text { algo insatisfecho }\end{array}$ & $\begin{array}{l}\text { Ni satisfecho ni } \\
\text { insatisfecho }\end{array}$ & $\begin{array}{l}\text { Muy satisfecho o } \\
\text { algo satisfecho }\end{array}$ \\
\hline \multirow{2}{*}{ Estrato 1} & $0,237^{* *}$ & $0,138^{* * *}$ & $-0,375^{\star * *}$ \\
\hline & $(0,098)$ & $(0,027)$ & $(0,121)$ \\
\hline \multirow{2}{*}{ Estrato 2} & $0,208^{* *}$ & $0,120^{* * *}$ & $-0,328^{* * *}$ \\
\hline & $(0,102)$ & $(0,028)$ & $(0,126)$ \\
\hline \multirow{2}{*}{ Estrato 3} & $0,227^{* *}$ & $0,126^{* * *}$ & $-0,353^{* * *}$ \\
\hline & $(0,102)$ & $(0,026)$ & $(0,123)$ \\
\hline \multirow{2}{*}{ Estrato 4} & $0,227^{*}$ & $0,105^{* * *}$ & $-0,331^{* * *}$ \\
\hline & $(0,118)$ & $(0,017)$ & $(0,128)$ \\
\hline \multirow{2}{*}{ Estrato 5} & $0,259^{*}$ & $0,089^{* * *}$ & $-0,348^{* * *}$ \\
\hline & $(0,143)$ & $(0,017)$ & $(0,133)$ \\
\hline Log-likelihood & & $-749,97$ & \\
\hline Observaciones & & 840 & \\
\hline \multicolumn{4}{|c|}{${ }^{*} \mathrm{p}<0,10 ;{ }^{* *} \mathrm{p}<0,05 ;{ }^{* * *} \mathrm{p}<0,01$} \\
\hline
\end{tabular}

Nota: los resultados provienen de un modelo cuya variable dependiente es el nivel de satisfacción de los individuos con respecto al servicio de salud, y la variable independiente el estrato socioeconómico de los entrevistados. Como variables de control se incluyeron variables dummy para los grupos de edad y de género. Desviaciones estándar robustas en paréntesis.

Fuente: elaboración propia con base en información de las encuestas Cómo vamos, 2011.

Estos resultados corroboran que, a pesar de los esfuerzos en cobertura y accesibilidad al sistema de salud, persisten aún ciertos niveles de desigualdad en la prestación del servicio en Barranquilla. Esto, por supuesto, es una oportunidad para la administración local de continuar mejorando la calidad del servicio. Queda la pregunta de ¿qué tan positivos o negativos son los resultados anteriores en términos relativos a otras ciudades? La Tabla 6 presenta la relación entre la satisfacción con el servicio de salud y el estrato socioeconómico para Cartagena, Bogotá y Medellín. 
Tabla 6. Efectos marginales de la condición socioeconómica sobre la satisfacción con la prestación de los servicios de salud en Cartagena, Bogotá y Medellín, 2011

\begin{tabular}{|c|c|c|c|}
\hline \multirow[b]{2}{*}{ Variable dependiente } & \multicolumn{3}{|c|}{ Cartagena } \\
\hline & $\begin{array}{l}\text { Muy insatisfecho o } \\
\text { algo insatisfecho }\end{array}$ & $\begin{array}{l}\text { Ni satisfecho ni } \\
\text { insatisfecho }\end{array}$ & $\begin{array}{l}\text { Muy satisfecho o } \\
\text { algo satisfecho }\end{array}$ \\
\hline \multirow{2}{*}{ Estrato 1} & $0,997^{* * *}$ & $-0,382^{* * *}$ & $-0,615^{* * *}$ \\
\hline & $(0,000)$ & $(0,120)$ & $(0,120)$ \\
\hline \multirow{2}{*}{ Estrato 2} & $0,998^{\star * *}$ & $-0,387^{* * *}$ & $-0,611^{* * *}$ \\
\hline & $(0,000)$ & $(0,141)$ & $(0,141)$ \\
\hline \multirow{2}{*}{ Estrato 3} & $0,993^{* * *}$ & $-0,464^{* * *}$ & $-0,529^{* * *}$ \\
\hline & $(0,001)$ & $(0,136)$ & $(0,136)$ \\
\hline \multirow{2}{*}{ Estrato 4} & $0,954^{* * *}$ & $-0,512^{* * *}$ & $-0,442^{* * *}$ \\
\hline & $(0,004)$ & $(0,118)$ & $(0,119)$ \\
\hline \multirow[t]{2}{*}{ Estrato 5} & $0,942^{* * *}$ & $-0,514^{* * *}$ & $-0,427^{* * *}$ \\
\hline & $(0,006)$ & $(0,117)$ & $(0,118)$ \\
\hline Log-likelihood & & $-506,29$ & \\
\hline Observaciones & & 503 & \\
\hline
\end{tabular}

\begin{tabular}{|c|c|c|c|}
\hline \multirow[b]{2}{*}{ Variable dependiente } & \multicolumn{3}{|c|}{ Bogotá } \\
\hline & $\begin{array}{l}\text { Muy insatisfecho o } \\
\text { algo insatisfecho }\end{array}$ & $\begin{array}{l}\text { Ni satisfecho ni } \\
\text { insatisfecho }\end{array}$ & $\begin{array}{l}\text { Muy satisfecho o } \\
\text { algo satisfecho }\end{array}$ \\
\hline \multirow{2}{*}{ Estrato 1} & $0,181^{\star *}$ & $0,063^{* * *}$ & $-0,244^{* * *}$ \\
\hline & $(0,071)$ & $(0,009)$ & $(0,073)$ \\
\hline \multirow{2}{*}{ Estrato 2} & $0,119^{* *}$ & $0,069^{* * *}$ & $-0,188^{\star * *}$ \\
\hline & $(0,050)$ & $(0,021)$ & $(0,070)$ \\
\hline \multirow{2}{*}{ Estrato 3} & $0,103^{* *}$ & $0,066^{* * *}$ & $-0,169^{* *}$ \\
\hline & $(0,046)$ & $(0,024)$ & $(0,069)$ \\
\hline \multirow{2}{*}{ Estrato 4} & $0,105^{*}$ & $0,056^{* * *}$ & $-0,161^{\star *}$ \\
\hline & $(0,058)$ & $(0,019)$ & $(0,076)$ \\
\hline \multirow[t]{2}{*}{ Estrato 5} & 0,096 & $0,049^{* *}$ & $-0,146^{*}$ \\
\hline & $(0,065)$ & $(0,020)$ & $(0,084)$ \\
\hline Log-likelihood & & $-1.003,56$ & \\
\hline Observaciones & & 1.020 & \\
\hline
\end{tabular}




\begin{tabular}{|c|c|c|c|}
\hline \multirow{2}{*}{ Variable dependiente } & \multicolumn{3}{|c|}{ Medellín } \\
\hline & $\begin{array}{l}\text { Muy insatisfecho o } \\
\text { algo insatisfecho }\end{array}$ & $\begin{array}{l}\text { Ni satisfecho ni } \\
\text { insatisfecho }\end{array}$ & $\begin{array}{c}\text { Muy satisfecho o } \\
\text { algo satisfecho }\end{array}$ \\
\hline \multirow{2}{*}{ Estrato 1} & 0,087 & $0,053^{*}$ & $-0,14$ \\
\hline & $(0,070)$ & $(0,031)$ & $(0,100)$ \\
\hline \multirow{2}{*}{ Estrato 2} & $0,139 * *$ & $0,077^{* * *}$ & $-0,216^{* *}$ \\
\hline & $(0,069)$ & $(0,024)$ & $(0,092)$ \\
\hline \multirow{2}{*}{ Estrato 3} & 0,087 & $0,061^{*}$ & $-0,148^{*}$ \\
\hline & $(0,056)$ & $(0,034)$ & $(0,089)$ \\
\hline \multirow{2}{*}{ Estrato 4} & 0,071 & 0,047 & $-0,118$ \\
\hline & $(0,062)$ & $(0,034)$ & $(0,095)$ \\
\hline \multirow[t]{2}{*}{ Estrato 5} & 0,078 & 0,049 & $-0,127$ \\
\hline & $(0,069)$ & $(0,032)$ & $(0,101)$ \\
\hline Log-likelihood & & $-856,09$ & \\
\hline Observaciones & & 924 & \\
\hline \multicolumn{4}{|c|}{${ }^{*} p<0,10 ;{ }^{* *} p<0,05 ;{ }^{* * *} p<0,01$} \\
\hline
\end{tabular}

Nota: los resultados provienen de un modelo cuya variable dependiente es el nivel de satisfacción de los individuos con respecto al servicio de salud, y la variable independiente el estrato socioeconómico de los entrevistados. Como variables de control se incluyeron variables dummy para los grupos de edad y de género. Desviaciones estándar robustas en paréntesis.

Fuente: elaboración propia con base en información de las encuestas Cómo vamos de las ciudades correspondientes, 2011.

Los resultados parecen ser muy claros en términos de la posible desigualdad socioeconómica en la prestación de los servicios de salud. Se puede observar que si se compara a Barranquilla con Cartagena, en esta última el problema de desigualdad parece ser más profundo ya que, por ejemplo, para las personas de estrato 1 , la probabilidad de sentirse insatisfechas con el servicio de salud aumenta en cerca de 1 pp cuando se compara con las de estrato 6, mientras que en Barranquilla para este mismo nivel socioeconómico la probabilidad era de tan solo 0,23 pp. Cuando se comparan los resultados de Barranquilla con los de Bogotá y Medellín, es posible observar cierto grado de ventaja de estas dos últimas, ya que los efectos marginales son menores, e incluso no-significativos, indicando que no existe una relación tan estrecha entre el nivel socioeconómico y la percepción de satisfacción con la prestación de los servicios de salud. El caso más evidente es el de Medellín, en donde es notoria la menor dependencia de la satisfacción con el nivel socioeconómico, lo que claramente podría interpretarse como una mayor equidad.

\section{Comentarios finales}

En Barranquilla, un sector particularmente beneficiado por las decisiones de política y el compromiso de las autoridades locales desde 2008, es el de la salud. La ciudad logró implementar y poner en marcha un nuevo modelo de prestación de servicios a través de la expansión y mejoramiento de su Red Pública 
Hospitalaria. El nuevo esquema cuenta ahora con modernos centros prioritarios (Pasos) y de mayor complejidad (Caminos), adicionales a los hospitales. Esto sin contar con el grupo de Caminantes, quienes recorren la ciudad recolectando información sobre el estado de salud de los habitantes, poniéndolos en contacto con la Red Pública Hospitalaria. Así las cosas, con más de veinte nuevas instituciones, totalmente dotadas a lo largo de la ciudad, se logró dar un vuelco a los indicadores de accesibilidad a los servicios de salud.

Los barranquilleros, los de mayores necesidades en particular, se encuentran ahora mucho más cerca no solo de los centros prioritarios, sino de los centros de mayor complejidad. Adicionalmente, el sector se ha venido modernizando y actualizando su infraestructura física, y ha venido reorganizando su situación financiera gracias a la entrada de la IPS Universitaria como operador de la Red Pública Hospitalaria. Esta es la oportunidad para que la ciudad aproveche los altos niveles de cobertura y accesibilidad, y se dedique a invertir mayores recursos en el mejoramiento en la calidad del servicio. El otro problema fundamental al que se deben dedicar esfuerzos adicionales es el de la inequidad en la prestación del servicio de salud. Esto se puede lograr a través de un sistema justo y transparente en donde la condición socioeconómica de los usuarios no los haga acreedores a un servicio diferencial por parte de los prestadores del servicio de salud del Distrito.

\section{Referencias bibliográficas}

Alcaldía de Barranquilla. Plan de desarrollo social, económico y de obras públicas 2008-2011. Barranquilla: Alcaldía de Barranquilla, 2008.

Apparicio, Philippe, Mohamed Abdelmajid, Mylène Riva y Richard Shearmur. "Comparing Alternative Approaches to Measuring the Geographical Accesibility of Urban Health Services: Distance Types and Aggregation-Error Issues». International Journal of Health Geographics, Vol. 7, 2008: 1-14.

Barranquilla Cómo vamos. Encuesta de percepción ciudadana de calidad de vida (2008, 2009, 2010, 2011). Barranquilla: Microdatos, 2008-2011.

Bogotá Cómo vamos. Encuesta de percepción ciudadana de calidad de vida (2008, 2009, 2010, 2011). Bogotá, D.C.: Microdatos, 2008-2011.

Brabyn, Lars, y Chris Skelly. «Modeling Population Access to New Zeland Public Hospitals». International Journal of Health Geographics, Vol. 1, 2002: 1-9.

Cartagena Cómo vamos. Encuesta de percepción ciudadana de calidad de vida (2008, 2009, 2010, 2011). Cartagena de Indias: Microdatos, 2008-2011.

Cepeda, Laura. «Los sures de Barranquilla: la distribución espacial de la pobreza». En La Economía de Barranquilla a comienzos del siglo XX, editado por Laura Cepeda, 1-40. Bogotá: Banco de la República - Colección de Economía Regional, 2013.

Comber, Alexis, Chris Brundsdon y Robert Radburn. «A Spatial Analysis of Variations in Health Access: Linking Geography, Socio-economic Status and Access Perceptions». International Journal of Health Geographics, Vol. 10, 2011: 1-11.

Christie, Stephen, y David Fone. «Equity of Access to Tertiary Hospitals in Wales: A Travel Time Analysis». Journal of Public Health Medicine, Vol. 25, n 4, 2003: 344-350. 
Doriwala, Hiral, y N.C. Shah. «GIS-Based Analysis of Facility Provision Accesible to Different Socio-Economic Groups in Surat City". World Applied Sciences Journal, Vol. 9, $\mathrm{n}^{\circ}$ 7, 2010: 740-745.

Garrocho, Carlos, y Juan Campos. «Un indicador de accesibilidad a unidades de servicios clave para ciudades mexicanas: fundamentos, diseño y aplicación». Economía, Sociedad y Territorio, Vol. VI, 2006: 1 - 60.

Gibson, John, Xianzheng Deng, Geua Boe-Gibson, Scott Rozelle y Gicun Huang. "Which Households are More Distant from Health Centers in Rural China? Evidence from a GIS Network Analysis». GeoJournal, Vol. 76, 2011: 245-255.

Gobernación del Atlántico. Evaluación de la suficiencia de la red prestadora de servicios de salud en el departamento del Atlántico y distrito de Barranquilla. Barranquilla: Gobernación de Barranquilla, 2010.

Guagliardo, Mark. «Spatial Accesibility of Primary Care: Concepts, Methods and Challenges». International Journal of Health Geographics, Vol. 3, 2004: 1-13.

Gulliford, Martin, Jose Figueron-Muñoz, Myfamwy Morgan, David Hughes, Barry Gibson, Roger Beech y Meryl Hudson. "What 'Access to Health Care' Mean?». Journal of Health Services Research and Policy, Vol. 7, 2002: 186-188.

Higgs, Gary "A Literature Review of the Use of GIS-Based Measures of Access to Health Care services». Health Services \& Outcomes Reserch Methodology, Vol. 5, $\mathrm{n}^{\circ}$ 2, 2004: 119-139.

Hossain, Monir, y James Laditka. «Using Hospitalization for Ambulatory Care Sensitive Conditions to Measure Access to Primary Health Care: An Application of Spatial Structural Equation Modeling». International Journal of Health Geographics, Vol. 8, 2009: 1-14.

Instituto Geográfico Agustín Codazzi (IGAC). Información cartográfica de la ciudad de Barranquilla. Bogotá D. C.: IGAC, s/f.

IPS Universitaria. Información sobre la Red Pública Hospitalaria de Barranquilla (2013). Barranquilla: Microdatos: 2008, 2013.

Luo, Wei, y Fahui Wang. «Measures of Spatial Accesibility to Health Care in a GIS Environment: Synthesis and a Case Study in the Chicago Region». Environment and Planning B: Planning and Design, Vol. 30, 2003: 865-884.

Medellín Cómo vamos. Encuesta de percepción ciudadana de calidad de vida (2008, 2009, 2010, 2011). Medellín: Microdatos, 2008-2011.

Orozco, Antonio. La salud en Barranquilla y Cartagena: evolución y percepción. Barranquilla: Mimeo, Banco de la República, 2013.

Penchasky, Roy, y William Thomas. «The Concept of Access. Definition and Relationship to Consumer Satisfaction». Medical Care, Vol. XIX, 1981: 127-140.

Rosero-Bixby, Luis. "Spatial Access to Health Care in Costa Rica and its Equity: A GIS-based Study». Social Science \& Medicine, Vol. 58, n 7, 2004: 1.271-1.284.

Unal, Eda, Susan Chen y Brigitte Waldorfd. «Spatial Accesibility of Health care in Indiana». Working Paper (Department of Agricultural Economics, Purdue Universtity), $n^{\circ}$ 07-07, (abril 2007): 1-26. 


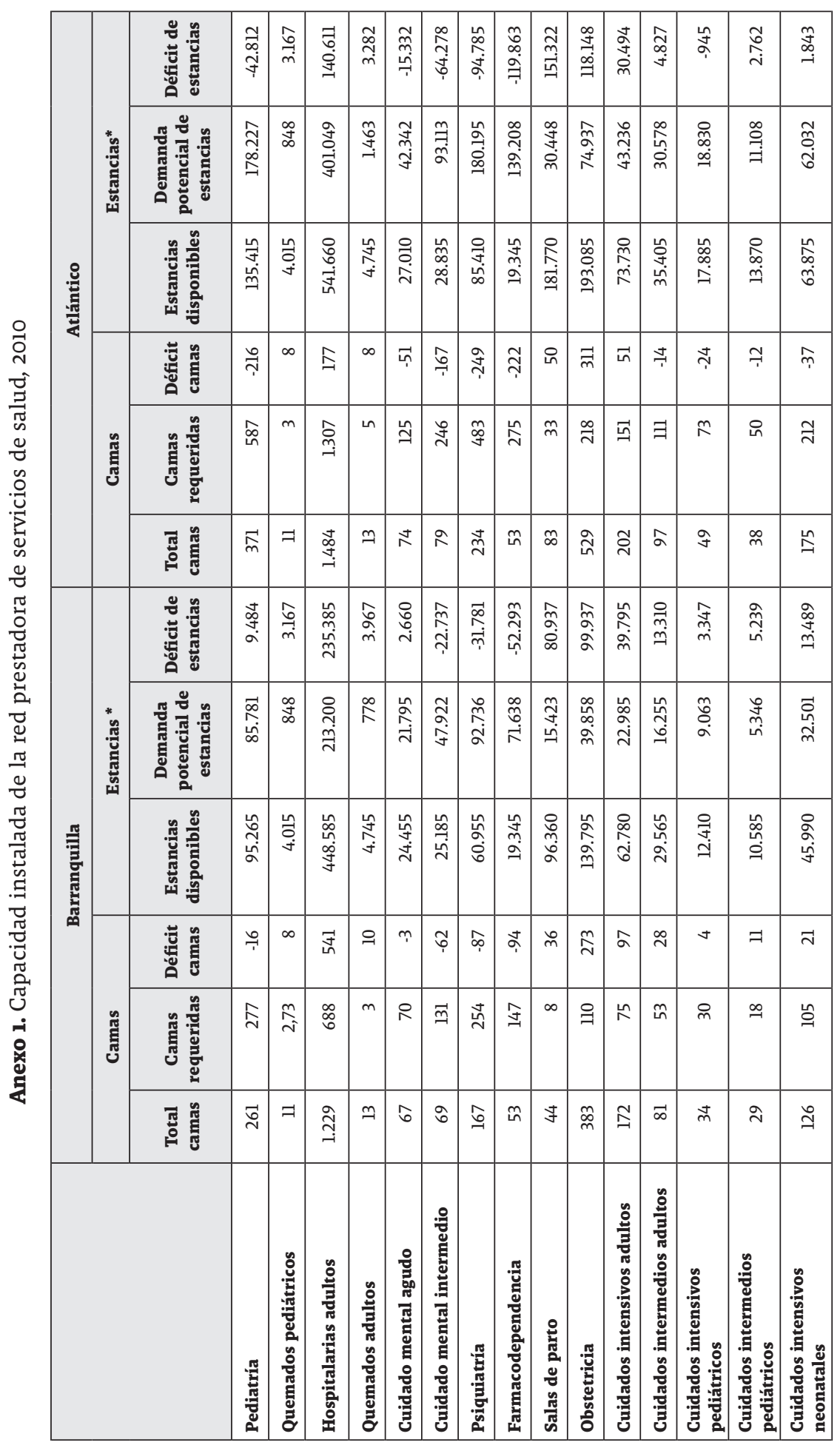




\begin{tabular}{|c|c|c|c|c|c|c|c|c|c|}
\hline \multirow{6}{*}{ 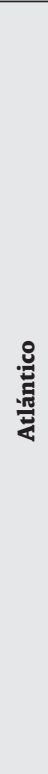 } & & 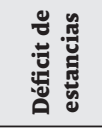 & $\begin{array}{l}\stackrel{\circ}{\circ} \\
\stackrel{\sim}{\infty} \\
\stackrel{\infty}{\infty}\end{array}$ & 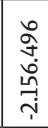 & 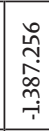 & 京 & 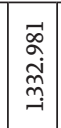 & 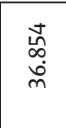 & \multirow{13}{*}{ 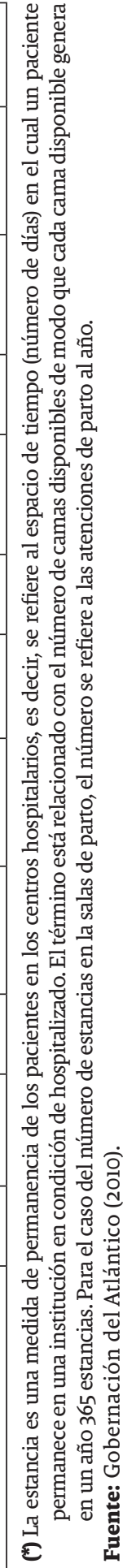 } \\
\hline & 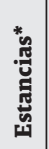 & 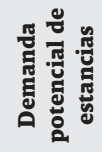 & 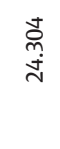 & 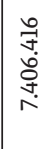 & 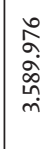 & 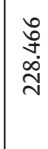 & 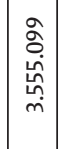 & $\begin{array}{l}\stackrel{2}{\circ} \\
\stackrel{1}{I}\end{array}$ & \\
\hline & & 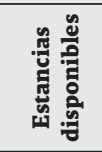 & ڤ્̀ & $\begin{array}{l}\text { ָे } \\
\stackrel{\alpha}{\alpha} \\
\text { ஸ் }\end{array}$ & $\begin{array}{l}\stackrel{\text { N }}{\text { ì }} \\
\text { ì }\end{array}$ & $\begin{array}{l}\underset{N}{\infty} \\
\infty \\
\stackrel{\tilde{N}}{\sim}\end{array}$ & $\begin{array}{c}0 \\
0 \\
0 \\
\infty \\
\infty \\
\infty \\
\dot{j} \\
\dot{j}\end{array}$ & $\begin{array}{l}\text { ర్రి } \\
\text { ป் }\end{array}$ & \\
\hline & & 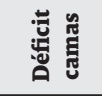 & in & 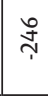 & $\underset{7}{~}$ & $m$ & $\vec{n}$ & $\infty$ & \\
\hline & 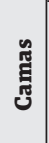 & 总 & $\infty$ & $\underset{\infty}{\mathbb{N}}$ & જे & $\widetilde{\Omega}$ & $\mathcal{F}$ & ঃ & \\
\hline & & 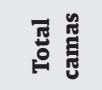 & $\stackrel{0}{J}$ & $\overrightarrow{\tilde{\sigma}}$ & $\stackrel{m}{m}$ & $\stackrel{\stackrel{一}{a ~}}{ }$ & a & $\stackrel{\circ}{\circ}$ & \\
\hline \multirow{6}{*}{ 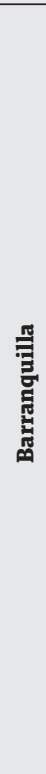 } & \multirow{3}{*}{ 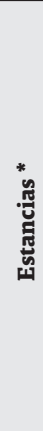 } & 窇 & $\begin{array}{l}\vec{D} \\
\text { مे } \\
\text { ñ }\end{array}$ & $\begin{array}{l}\stackrel{\infty}{\alpha} \\
\hat{n} \\
\hat{n}\end{array}$ & 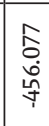 & $\underset{\substack{\infty \\
\stackrel{\infty}{\infty}}}{\stackrel{\infty}{\infty}}$ & 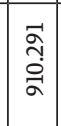 & $\begin{array}{l}\hat{a} \\
\vec{m} \\
\vec{m}\end{array}$ & \\
\hline & & 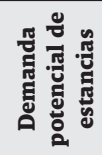 & 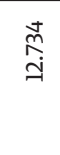 & 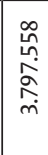 & 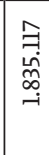 & 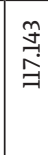 & 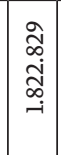 & 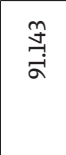 & \\
\hline & & 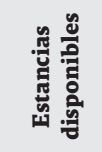 & $\begin{array}{l}\stackrel{\sim}{\tilde{q}} \\
\stackrel{\vartheta}{q}\end{array}$ & $\begin{array}{l}8 \\
\stackrel{8}{0} \\
\dot{0} \\
\dot{m} \\
\dot{m}\end{array}$ & \begin{tabular}{l}
$q$ \\
\multirow{0}{0}{} \\
$\stackrel{i}{0}$ \\
$\stackrel{m}{-1}$
\end{tabular} & 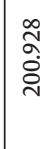 & 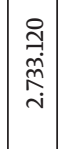 & $\begin{array}{l}\text { ণ̦ } \\
\text { త్ }\end{array}$ & \\
\hline & \multirow{3}{*}{ 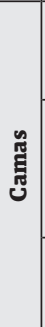 } & 䔍 & 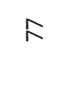 & $\stackrel{?}{\uparrow}$ & $\stackrel{\sim}{*}$ & $\hat{\sigma}$ & $\stackrel{L}{m}$ & 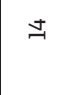 & \\
\hline & & 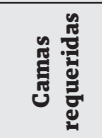 & F & 岇 & $\stackrel{\text { q }}{N}$ & t & A & $\stackrel{f}{\mathcal{F}}$ & \\
\hline & & 胥 & 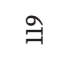 & $\stackrel{\otimes}{\infty}$ & $\overline{\text { ปี }}$ & 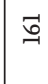 & in & เి & \\
\hline & & & 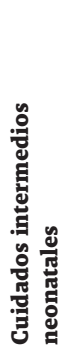 & 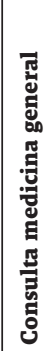 & 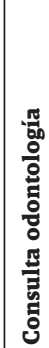 & 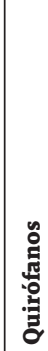 & 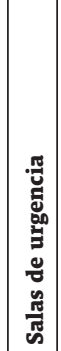 & 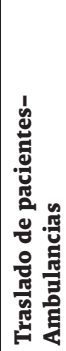 & \\
\hline
\end{tabular}


Anexo 2. Cambios en la distribución de la accesibilidad a la red pública de salud

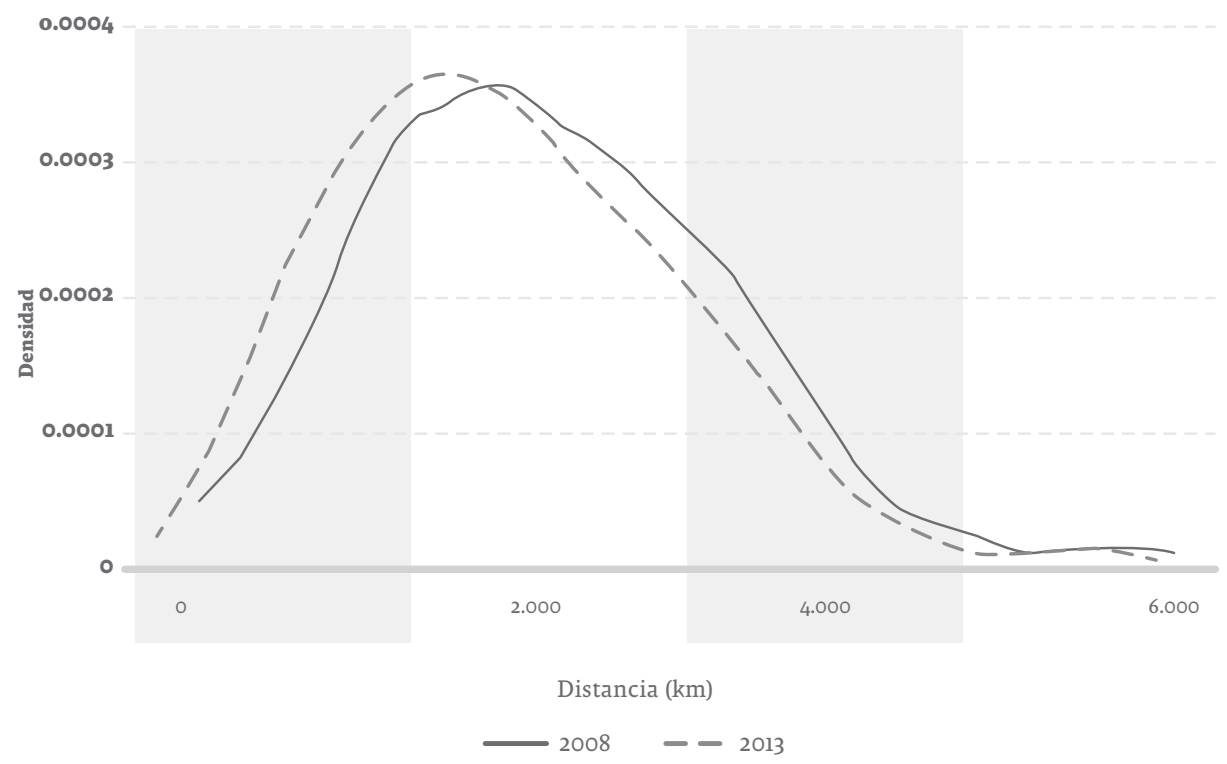

Fuente: elaboración propia con base en información de la IPS Universitaria (2013). 